\title{
Local rainbow colorings
}

\author{
Noga Alon* AND Ido Ben-Eliezer ${ }^{\dagger}$
}

\begin{abstract}
Given a graph $H$, we denote by $C(n, H)$ the minimum number $k$ such that the following holds. There are $n$ colorings of $E\left(K_{n}\right)$ with $k$-colors, each associated with one of the vertices of $K_{n}$, such that for every copy $T$ of $H$ in $K_{n}$, at least one of the colorings that are associated with $V(T)$ assigns distinct colors to all the edges of $E(T)$.

We characterize the set of all graphs $H$ for which $C(n, H)$ is bounded by some absolute constant $c(H)$, prove a general upper bound and obtain lower and upper bounds for several graphs of special interest. A special case of our results partially answers an extremal question of Karchmer and Wigderson motivated by the investigation of the computational power of span programs.
\end{abstract}

\section{Introduction}

Consider the following question, motivated by an extremal problem suggested by Karchmer and Wigderson, see [6]. Given a fixed graph $H$, let $C(n, H)$ denote the minimum number $k$ such that there is a set of $n$ colorings $\left\{f_{v}: E\left(K_{n}\right) \rightarrow[k]: v \in V\left(K_{n}\right)\right\}$, with the following property. For every copy $T$ of $H$ in $K_{n}$, there is a vertex $u \in V(T)$ so that $f_{u}$ is a rainbow coloring of $E(T)$, that is, no two edges of $T$ get the same color by $f_{u}$. A set of colorings that satisfies this condition is called an $(n, H)$-local coloring. Determine or estimate the function $C(n, H)$. Note that each coloring in the set above does not have to be a proper edge coloring.

For example, if $T$ is a path with 2 edges, two colors are sufficient for every $n$. Indeed, simply define $f_{v}(u w)=1$ if $v \in\{u, w\}$, and $f_{v}(u w)=2$ otherwise. Obviously, this is a legal local coloring, and therefore we conclude that $C\left(n, P_{2}\right)=2$ for all $n \geq 3$. As we show in this short paper, for almost every graph $T$, every $(n, T)$-local coloring requires a number of colors that grows as $n$ grows. In fact, even the path $P_{3}$ of 3 edges requires more than a constant number of colors.

${ }^{*}$ Research supported in part by an ERC Advanced grant, by a USA-Israeli BSF grant and by NSF grant No. DMS-0835373.

${ }^{\dagger}$ Research supported in part by the Dan David fellowship for PhD students and by an ERC advanced grant. 
Motivation. Karchmer and Wigderson [5] defined the Span Program computational model. This is a linear algebra computational model. The program is defined by a set of vectors $A$ over $G F_{2}$, where each vector is marked with a literal. Every input of the program corresponds to the subset of vectors of $A$ whose marking literals have value 1 in the given input, and the input is accepted if and only if this subset spans some fixed vector, say the all-ones vector. It turns out that this model is stronger than other known models like Switching Networks and DeMorgan Formulas (see [4]), and therefore lower bounds for the minimum possible size of Span Programs that compute explicit Boolean functions are desirable.

The Fusion Method of Karchmer and Wigderson [5, 6] enables one to prove lower bounds for this model using certain extremal results. With this motivation in mind, Wigderson suggested in [6] three problems. One of them is the following (also suggested as a research problem in [4], Chapter 16, Problem 9).

Problem 1.1. Let $k$ be the minimum number for which the following holds. There exist $n$ colorings $c_{1}, c_{2}, \ldots, c_{n}$ of the $n$-cube $\{0,1\}^{n}$ in $k$ colors such that for every triple of distinct vectors $x, y, z$ there is a coordinate $i$ on which not all three vectors agree and the three colors $c_{i}(x), c_{i}(y), c_{i}(z)$ are pairwise distinct. Determine or estimate the smallest number $k$ for which such a collection of colorings exist.

In [5] it is shown that $k$ does have to grow with $n$, proving that it is at least $\Omega\left(\frac{\log \log ^{*} n}{\log \log \log ^{*} n}\right)$, where $\log ^{*} n$ is the minimum number $m$ so that starting with $n$ one gets a number that does not exceed 1 by iteratively applying the function $\log _{2}(x) \quad m$ times. As a special case of our results we obtain a far better lower bound, proving that for the path with 3 edges $P_{3}$, every $\left(n, P_{3}\right)$ local coloring requires $\Omega\left(\left(\frac{\log n}{\log \log n}\right)^{1 / 4}\right)$ colors. It is easy to see that this lower bound holds for the number of required colors in the problem above as well, even if one considers only vectors of Hamming weight 2. Indeed, colorings of all vectors with Hamming weight 2 can be interpreted as colorings of all edges of the complete graph in the obvious way, and if these colorings satisfy the requirement in the problem for any triple of vectors of Hamming weight 2 , then the same set of colorings forms an $(n, H)$-local coloring for all graphs $H$ with exactly three edges, including $P_{3}$. Unfortunately, it does not seem that this improved bound yields any new results for span programs.

Our results. Our first result characterizes the set of all graphs $H$ for which $C(n, H)$ is bounded by some absolute constant that depends only on $H$. To this end, we define 2-locally large graphs, and show that $C(n, H) \leq c(H)$ 
for every $n$ if and only if $H$ is not 2-locally large. This implies the following somewhat surprising corollary dealing with graphs that are not 2-locally large.

Theorem 1.2. For a fixed graph $H$ there is a constant $c(H)$ so that $C(n, H) \leq c(H)$ for every $n$ if and only if $H$ contains at most 3 edges and $\mathrm{H}$ is neither $P_{3}$ nor $P_{3}$ together with any number of isolated vertices. Moreover, in all these cases $C(n, H) \leq 5$ for every $n$.

We next consider upper bounds. We start with a simple general upper bound for every fixed graph $H$, obtained by applying the local lemma. This shows that for every fixed graph $H, C(n, H)$ is sublinear in $n$.

Theorem 1.3. Let $H$ be a fixed graph with $r$ vertices. Then $C(n, H)=$ $O\left(n^{\frac{r-2}{r}} \cdot r^{4}\right)$.

Note that the most difficult case here is when $H$ is a complete graph on $r$ vertices, though this general approach improves the bound for sparser graphs only by a constant that depends on $r$.

We also provide a simple explicit construction for the case $H=P_{3}$. This construction is summarized in the following proposition.

Proposition 1.4. There is an explicit construction showing that $C\left(n, P_{3}\right) \leq$ $2\lceil\sqrt{n}\rceil$.

In the last part of this work we obtain lower bounds for several graphs, including a $(\log n)^{\Omega(1)}$ lower bound for $C(n, H)$ for $H=P_{3}$, for any star with $t \geq 4$ leaves, and for any graph consisting of $t \geq 4$ independent edges. This implies a similar lower bound for any fixed graph with sufficiently many edges.

Theorem 1.5. Let $P_{t}$ denote the path with $t$ edges, $I_{t}$ the graph consisting of $t$ independent edges and $S_{t}$ the star with $t$ edges. The following lower bounds hold.

1. $C\left(n, P_{3}\right)=\Omega\left(\left(\frac{\log n}{\log \log n}\right)^{1 / 4}\right)$.

2. $C\left(n, I_{4}\right)=\Omega\left(n^{1 / 6}\right)$ and $C\left(n, I_{t}\right)=\Omega\left(n^{1 / 4}\right)$ for $t \geq 5$.

3. $C\left(n, S_{4}\right)=\Omega\left(n^{1 / 4}\right)$ and $C\left(n, S_{t}\right)=\Omega\left(n^{1 / 3}\right)$ for $t \geq 5$.

4. $C\left(n, P_{7}\right), C\left(n, P_{8}\right)=\Omega\left(n^{1 / 6}\right)$ and $C\left(n, P_{t}\right)=\Omega\left(n^{1 / 4}\right)$ for any fixed $t \geq 9$.

A simple modification of our proof also yields a $(\log n)^{\Omega(1)}$ lower bound for $C\left(n, P_{k}\right)$ for each $k \in\{4,5,6\}$. Observe that if $H^{\prime} \subset H$ is a subgraph of $H$ on the same set of vertices then every lower bound for $C\left(n, H^{\prime}\right)$ implies 
the same lower bound for $C(n, H)$. As every large enough graph $H$ (in terms of the number of edges) contains a star with 4 edges or 4 independent edges, and as it is possible to deal with isolated vertices in these cases, one can prove an $n^{\Omega(1)}$ lower bound for any fixed graph with sufficiently many edges. This is stated in the following.

Theorem 1.6. For any graph $H$ with at least 13 edges there is a constant $b=b(H)>0$ so that $C(n, H)=\Omega\left(n^{b}\right)$.

\section{Preliminaries}

Let $K_{n}$ denote the complete graph on $n$ vertices, and $K_{n}^{(t)}$ the hypergraph containing all subsets of size $t$ of $[n]$. In the course of the proofs we will apply the following hypergraph Ramsey result for 3-uniform hypergraphs, due to Erdős and Rado [3].

Theorem 2.1. There is an absolute constant c such that if $n>2^{2^{c k \log (k) r}}$ the following holds. For every $k$-coloring of $E\left(K_{n}^{(3)}\right)$ there is a set $T \subseteq V\left(K_{n}^{(3)}\right)$ of size $r$, such that $\left\{A \in E\left(K_{n}^{(3)}\right): A \subset T\right\}$ is monochromatic.

Given a graph $G$, we denote by $\Delta(G)$ the maximum degree of a vertex in $G$. A proper edge coloring of a graph $G$ is a mapping $\varphi: E(G) \rightarrow[k]$ such that every two adjacent edges get distinct colors. The edge chromatic number (or chromatic index) of any simple graph $G$ is the minimum $k$ for which such a coloring exists. Vizing's Theorem (see, e.g., [2]) states that the edge chromatic number of any simple graph $G$ is either $\Delta(G)$ or $\Delta(G)+1$.

\section{Graphs requiring a bounded number of colors}

Our goal in this section is to prove Theorem 1.2. We start with some auxiliary definitions.

Let $H$ be a fixed graph on $r$ vertices, and let $\sigma: V(H) \rightarrow[r]$ be a permutation. Roughly speaking, $H$ is 2-locally large if for every vertex $v$ there are two edges that look the same with respect to $\sigma$ and $v$. More formally, for every vertex $v \in V(H)$, define the following five types of edges.

1. $T_{v}^{1}=\{(u, v) \in E(H): \sigma(u)>\sigma(v)\}$.

2. $T_{v}^{2}=\{(u, v) \in E(H): \sigma(u)<\sigma(v)\}$.

3. $T_{v}^{3}=\left\{\left(u_{1}, u_{2}\right) \in E(H): \sigma(v)<\sigma\left(u_{1}\right)<\sigma\left(u_{2}\right)\right\}$.

4. $T_{v}^{4}=\left\{\left(u_{1}, u_{2}\right) \in E(H): \sigma\left(u_{1}\right)<\sigma(v)<\sigma\left(u_{2}\right)\right\}$.

5. $T_{v}^{5}=\left\{\left(u_{1}, u_{2}\right) \in E(H): \sigma\left(u_{1}\right)<\sigma\left(u_{2}\right)<\sigma(v)\right\}$. 
Thus there are two types of edges that are incident to $v$, backward edges and forward edges (with respect to $\sigma$ ). In addition, there are three types of non-incident edges, again with respect to $\sigma$. There are edges before $v$, edges after $v$, and edges that cross $v$.

Definition. A graph $H$ on $r$ vertices is 2-locally large if there is a permutation $\sigma: V(H) \rightarrow[r]$ such that for every $v \in V(H)$, at least one of the sets $T_{v}^{1}, \ldots, T_{v}^{5}$ has at least two elements.

We can now state the main result of this section.

Theorem 3.1. Let $H$ be a fixed graph. Then $C(n, H) \leq c(H)$ for every $n$ if and only if $H$ is not 2-locally large. In this case $C(n, H) \leq 5$ for all $n$.

Before presenting the proof of this theorem, we turn to list the graphs which are not 2-locally large. It is obvious that every graph with at least 6 edges is 2-locally large. A path of length 3, denoted by $x y z w$ is also 2-locally large by letting $\sigma(x)<\sigma(z)<\sigma(y)<\sigma(w)$. It is also easy to prove by inspection that every graph with at most 3 edges besides the path $P_{3}$ is not 2-locally large. We next claim that actually every graph with at least 4 edges is 2-locally large.

Proposition 3.2. Let $H$ be a graph with at least 4 edges. Then $H$ is 2-locally large.

Proof. It is sufficient to prove the assertion for graphs with exactly 4 edges and no isolated vertices, as the property of being 2-locally large is maintained under addition of edges or isolated vertices. Consider the following cases.

- $H$ has 4 independent edges, denoted by $\left(u_{1}, u_{2}\right),\left(u_{3}, u_{4}\right),\left(u_{5}, u_{6}\right),\left(u_{7}\right.$, $\left.u_{8}\right)$. In this case let $\sigma\left(u_{i}\right)<\sigma\left(u_{j}\right)$ for every $i<j$ and we are done.

- $H$ is the union of two paths with two edges each. Denote these paths by $u_{1} u_{2} u_{3}$ and $v_{1} v_{2} v_{3}$. Then by taking $\sigma\left(u_{i}\right)<\sigma\left(v_{j}\right)$ for every $1 \leq i, j \leq 3$, we get that every vertex has two indistinguishable edges.

- $H$ is the union of a path with two edges, denoted by $x y z$ with 2 independent edges, denoted by $\left(u_{1}, u_{2}\right)$ and $\left(u_{3}, u_{4}\right)$. In this case let $\sigma(x)<\sigma(y)<\sigma(z)<\sigma\left(u_{1}\right)<\sigma\left(u_{2}\right)<\sigma\left(u_{3}\right)<\sigma\left(u_{4}\right)$ and we are done.

- $H$ contains a connected component with 3 edges and a single independent edge $\left(u_{1}, u_{2}\right)$. In this case the connected component is either a path of length 3 (which is 2-locally large), a triangle or a star with 3 leaves. In the last two cases we let $\sigma\left(u_{1}\right)=1, \sigma\left(u_{2}\right)=|V(H)|$ and by symmetry it is easy to see that for every mapping of the vertices of the connected component and for every vertex $v$ we have $\left|\left(T_{v}^{i}\right) \cap E(H)\right|>1$ for some $i$. 
- $H$ is connected (and has 4 edges). In this case $H$ contains a path of length 3 or a star with 4 leaves, and in the last case every embedding will suffice.

Proof of Theorem 1.2. By Theorem 3.1 $C(n, H)$ grows as $n$ grows if and only if $H$ is 2-locally large, and otherwise it is bounded by 5 . By Proposition 3.2 these graphs are exactly all graphs with at least 4 edges and $P_{3}$. Theorem 1.2 follows.

Proof of Theorem 3.1. We start by proving that for every graph $H$ that is not 2-locally large, $C(n, H)$ is bounded by 5 . Indeed, let $K_{n}$ be the complete graph on $n$ vertices, and fix an arbitrary mapping $\sigma: V\left(K_{n}\right) \rightarrow[n]$. For every vertex $v \in K_{n}$, we let $f_{v}(x y)=i$ if and only if $x y \in T_{v}^{i}$.

Let $T$ be a subgraph of $K_{n}$ isomorphic to $H$. Since $T$ is not 2-locally large, there is a vertex $v \in V(T)$ such that $\left|\left(T_{v}^{i}\right) \cap E(H)\right| \leq 1$ for every $i$, and hence $f_{v}\left(e_{1}\right) \neq f_{v}\left(e_{2}\right)$ for every $e_{1}, e_{2} \in E(T)$, as required.

Suppose now that $H$ is 2-locally large. Let $\sigma: V\left(K_{n}\right) \rightarrow[n]$ be an arbitrary bijection, and let $f_{1}, \ldots, f_{n}: E(G) \rightarrow[k]$ be $n$ colorings, one for each vertex. We define a coloring of $K_{n}^{(3)}$, the complete 3 -uniform hypergraph, which is defined with a slight abuse of notation on the same set of vertices as $K_{n}$. For every three vertices $u_{1}, u_{2}, u_{3} \in V\left(K_{n}\right)$, with $\sigma\left(u_{1}\right)<\sigma\left(u_{2}\right)<\sigma\left(u_{3}\right)$ we define the coloring of the hyperedge $\left(u_{1}, u_{2}, u_{3}\right)$ as the following ordered nine-tuple:

$$
\begin{aligned}
& {\left[f_{u_{1}}\left(u_{1} u_{2}\right), f_{u_{1}}\left(u_{1} u_{3}\right), f_{u_{1}}\left(u_{2} u_{3}\right), f_{u_{2}}\left(u_{1} u_{2}\right), f_{u_{2}}\left(u_{1} u_{3}\right), f_{u_{2}}\left(u_{2} u_{3}\right),\right.} \\
& \left.f_{u_{3}}\left(u_{1} u_{2}\right), f_{u_{3}}\left(u_{1} u_{3}\right), f_{u_{3}}\left(u_{2} u_{3}\right)\right] .
\end{aligned}
$$

This is a coloring of $E\left(K_{n}^{(3)}\right)$ with $k^{9}$ colors. By Theorem 2.1, if $n>$ $2^{2^{9 c k} k^{\log k r}}$, there is a monochromatic set of vertices $Q$ of size $r$ (namely, all 3 -edges that are contained in $Q$ have the same color). Consider a copy $T$ of $H$ in the vertices of $Q$ such that for every vertex $v$ in $T$, there is $1 \leq i \leq 5$ such that $\left|\left(T_{v}^{i}\right) \cap E(H)\right|>1$ (observe that there is such a copy since $T$ is 2-locally large).

We complete the proof by showing that $T$ does not admit a rainbow coloring $f_{v}$ for any $v \in V(T)$. Take a vertex $v \in V(T)$. Let $e_{1}, e_{2} \in E(T)$ such that $e_{1}, e_{2} \in T_{v}^{i}$ for some $1 \leq i \leq 5$. Since the two hyperedges that contain $v, e_{1}$ and $v, e_{2}$ are colored the same, we conclude that $f_{v}\left(e_{1}\right)=f_{v}\left(e_{2}\right)$. Therefore, every vertex $v \in T$ has two edges which get the same color, and we conclude that $K_{n}$ does not admit an $(n, H)$-local coloring by $k$ colors, as required. 
Note that the above proof shows that for every fixed $H$ which is 2-locally large, $C(n, H) \geq c_{H}\left(\frac{\log \log n}{\log \log \log n}\right)^{1 / 9}$.

It is worth noting that our approach cannot give lower bounds better than $\Omega\left((\log \log n)^{1 / 9}\right)$, even if the correct behavior of the Ramsey number for 3-uniform hypergraphs is the known lower bound for it rather than the known upper bound.

\section{Upper bounds}

In this section we prove a simple upper bound on $C(n, H)$ for every $H$, and also provide an explicit construction for the specific case of $H=P_{3}$.

Proof of Theorem 1.3. Clearly it is enough to prove the theorem for the case that $H$ is a complete graph on $r$ vertices, as if $f_{v}$ assigns pairwise distinct colors to the set of edges of $K_{r}$, it also gives distinct colors for every subgraph of $K_{r}$.

Let $k=n^{\frac{r-2}{r}} \cdot r^{4}$. For every $v \in V(G), e \in E(G)$, let $f_{v}(e)$ be uniformly distributed among the $k$ possible colors, independently of the other choices. We will prove that this random coloring is good with a positive probability by applying the symmetric case of the Local Lemma (see, e.g., [1], Chapter 5).

Indeed, given a fixed graph $T$ that is isomorphic to $H$, and a vertex $v \in V(T)$, the probability that two edges in $T$ get the same color in $f_{v}$ is clearly bounded by

$$
\frac{\left(\begin{array}{l}
r \\
2
\end{array}\right) \cdot\left(\left(\begin{array}{l}
r \\
2
\end{array}\right)-1\right)}{2 k} \leq \frac{r^{4}}{8 k} .
$$

Denote by $A(T)$ the event that all the colorings $f_{v}$, for every $v \in V(T)$, do not assign pairwise distinct colors to all edges in $E(T)$. Since the colorings are chosen independently, we conclude that $\operatorname{Pr}[A(T)] \leq\left(\frac{r^{4}}{8 k}\right)^{r}$.

Observe that each event $A\left(T_{1}\right)$ is mutually independent of all other events $A\left(T_{2}\right)$ besides those for which $T_{1}, T_{2}$ share at least one edge, and therefore at least two vertices. Thus, for every $T, A(T)$ is independent of all but at most $\left(\begin{array}{l}n-2 \\ r-2\end{array}\right)<n^{r-2}$ other events. We have

$$
n^{r-2} \cdot\left(\frac{r^{4}}{8 k}\right)^{r} \cdot e<1 .
$$

Hence, by the Local Lemma (see, e.g., [1], Chapter 5), we get that with positive probability none of the events $A(T)$ occurs, and hence a good coloring exists. 
We next show an explicit construction for the case of $H=P_{3}$. This explicit construction is slightly better than the randomized one (by a constant factor).

Proof of Proposition 1.4. We will find a set of $n$ colorings of the edges of $K_{n}$ by $2\lceil\sqrt{n}\rceil$ colors, such that in each copy of $P_{3}$ at least two of the vertices assign distinct colors to all edges. By Vizing's theorem, $K_{n}$ admits an $n$-edge coloring $\varphi$, where no two adjacent edges get the same color. Denote these colors by $1,2, \ldots, n$, and let $s=\lceil\sqrt{n}\rceil$. Clearly, for every $1 \leq q^{\prime}<q^{\prime \prime} \leq n$, we have $\left\lceil q^{\prime} / s\right\rceil \neq\left\lceil q^{\prime \prime} / s\right\rceil$ or $q^{\prime} \bmod s \neq q^{\prime \prime} \bmod s$.

We next define $f_{v}(a b)$ for every $v \in V\left(K_{n}\right)$ by a pair $\langle x, y\rangle$ with $x \in$ $\{0,1\}$ and $y \in[s]=\{1,2, \ldots, s\}$ as follows. The first coordinate determines whether $a b$ is incident with $v$ or not (that is, $x=1$ if either $a=v$ or $b=v$, and $x=0$ otherwise). Let $r=\varphi(a b)$. For the second coordinate, if $x=1$ we define the second coordinate of $f_{v}(a b)$ as $y=\lceil r / s\rceil$, and if $x=0$ we define it as $y=r \bmod s$.

Let $a b c d$ be a copy of $P_{3}$ in $K_{n}$. Then $f_{b}(a b), f_{b}(b c) \neq f_{b}(c d)$ and $f_{d}(a b), f_{d}(b c) \neq f_{d}(c d)$, as their first coordinates differ. Now, let $r_{1}=\varphi(a b)$ and $r_{2}=\varphi(b c)$. Since $\varphi$ is a legal edge coloring, $r_{1} \neq r_{2}$ and therefore $\left\lceil r_{1} / s\right\rceil \neq\left\lceil r_{2} / s\right\rceil$ or $r_{1} \bmod s \neq r_{2} \bmod s$. Therefore $f_{b}(a b) \neq f_{b}(b c)$ or $f_{d}(a b) \neq f_{d}(b c)$ and we get at least one good coloring of abcd. The same argument shows that also either $f_{a}$ or $f_{c}$ defines a good coloring of $a b c d$, and the proposition follows.

\section{Lower bounds}

In this section we obtain several lower bounds which are significantly better than the general lower bound that follows from the proofs of Theorems 1.2 and 3.1.

We start with a $(\log n)^{\Omega(1)}$ lower bound for $H=P_{3}$.

Proof of Item 1 in Theorem 1.5. Let $K_{n}$ be the complete graph on $n>$ $k^{\left(k^{2}+1\right)^{2}}+k^{2}+2$ vertices, and let $Y$ be an arbitrary set of vertices of size $k^{2}+1$ and $X=V\left(K_{n}\right) \backslash Y$. Fix a coloring $f_{v}$ of the edges of $K_{n}$ for every $v \in V\left(K_{n}\right)$. For every vertex $x \in X$, define a vector with $|Y|^{2}$ coordinates, by letting $x\left[u_{1}, u_{2}\right]=f_{u_{1}}\left(x u_{2}\right)$ for every $u_{1}, u_{2} \in Y$. By the pigeonhole principle, there are two vertices $x_{1}, x_{2} \in X$ such that the vector corresponding to $x_{1}$ equals that corresponding to $x_{2}$. By applying again the pigeonhole principle, there are two vertices $y_{1}, y_{2} \in Y$ such that $f_{x_{1}}\left(x_{2} y_{1}\right)=f_{x_{1}}\left(x_{2} y_{2}\right)$ and $f_{x_{2}}\left(x_{2} y_{1}\right)=f_{x_{2}}\left(x_{2} y_{2}\right)$. It follows that the path $x_{1} y_{1} x_{2} y_{2}$ does not have a rainbow coloring. Therefore, if there are good colorings $f_{1}, f_{2}, \ldots, f_{n}$ for $K_{n}$ then $n<k^{\left(k^{2}+1\right)^{2}}+k^{2}+2$ and hence $k=\Omega\left(\left(\frac{\log n}{\log \log n}\right)^{1 / 4}\right)$, as required. 
We proceed with an auxiliary lemma that will be used in proving lower bounds for the other cases.

Lemma 5.1. Let $\left\{f_{v}: v \in V\left(K_{2 n}^{(t)}\right)\right\}$ be a set of $2 n$ colorings of $V\left(K_{2 n}^{(t)}\right)$ with $k$ colors. Suppose that for every $e \in E\left(K_{2 n}^{(t)}\right)$ there is a vertex $v \in e$ such that $f_{v}$ assigns distinct colors to all the vertices of $e$.

1. If $t=4$ then $k \geq(1-o(1)) n^{1 / 3}$.

2. If $t \geq 5$ then $k \geq\left(\frac{1}{2}-o(1)\right) n^{1 / 2}$.

Proof. Let $A, B \subseteq V\left(K_{2 n}^{(t)}\right)$ be a partition of the vertices into two disjoint sets of size $n$. For every $a \in A$, the vertices of $B$ are partitioned into at most $k$ sets according to their color in $f_{a}$. We proceed by counting the number of pairs of elements in $B$ that are colored the same by some vertex in $A$ (with multiplicities). By convexity, every vertex $a \in A$ contributes

$$
\sum_{i=1}^{k}\left(\begin{array}{c}
\left|f_{a}^{-1}(i)\right| \\
2
\end{array}\right) \geq k \cdot\left(\begin{array}{c}
n / k \\
2
\end{array}\right)=\frac{n^{2}}{2 k} \cdot(1-k / n) .
$$

Therefore, there is a pair of vertices $b^{\prime}, b^{\prime \prime} \in B$ that are colored the same by at least

$$
\frac{n^{3}}{2 k\left(\begin{array}{c}
|B| \\
2
\end{array}\right)} \cdot(1-k / n) \geq \frac{n}{k}-1
$$

members of $A$.

Suppose that $t=4$. If $k<n^{1 / 3}(1-o(1))$ we conclude that there is a set $A^{\prime} \subseteq A$ of size $k^{2}+1$ and two elements $b^{\prime}, b^{\prime \prime} \in B$ such that for every $a \in A^{\prime}$ we have $f_{a}\left(b^{\prime}\right)=f_{a}\left(b^{\prime \prime}\right)$. By the pigeonhole principle, among the elements of $A^{\prime}$ there is a set $A^{\prime \prime}$ of size $k+1$ whose members are mapped to the same value by $f_{b^{\prime}}$. Similarly, there are two elements from $A^{\prime \prime}$, denoted by $a^{*}$ and $a^{* *}$ that are mapped to the same value by $f_{b^{\prime \prime}}$, and we conclude that the set $b^{\prime}, b^{\prime \prime}, a^{*}, a^{* *}$ does not admit a good coloring, and therefore $k \geq n^{1 / 3}(1-o(1))$ as required.

Suppose, now, that $t \geq 5$ and $k<\frac{1}{2} \cdot n^{1 / 2}(1-o(1))$. Then there is a set $A^{\prime}$ of size $4 k+t+1$ and two elements $b^{\prime}, b^{\prime \prime} \in B$ such that for every $a \in A^{\prime}$ we have $f_{a}\left(b^{\prime}\right)=f_{a}\left(b^{\prime \prime}\right)$. Applying iteratively the pigeonhole principle, we get that there is a set $X^{\prime}$ of $\frac{2 k+t}{3}$ triplets of elements in $A^{\prime}$, where the members of each triple have the same $f_{b^{\prime}}$ image, and there is a set $X^{\prime \prime}$ of $\frac{2 k+t}{3}$ triplets of elements in $A^{\prime}$ so that the members of each triple have the same $f_{b^{\prime \prime}}$ image. Therefore there are two triplets, one from $X^{\prime}$ and one from $X^{\prime \prime}$ that intersect, denote them by $\left(x_{1}^{\prime}, x_{2}^{\prime}, x_{3}^{\prime}\right)$ (from $\left.X^{\prime}\right)$ and $\left(x_{1}^{\prime}, x_{2}^{\prime \prime}, x_{3}^{\prime \prime}\right)$ (from $\left.X^{\prime \prime}\right)$. 
Without loss of generality assume that $x_{2}^{\prime} \neq x_{2}^{\prime \prime}$. If $t=5$, we get that the set $b^{\prime}, b^{\prime \prime}, x_{1}^{\prime}, x_{2}^{\prime}, x_{2}^{\prime \prime}$ does not admit a good coloring and we are done. If $t>5$, add to this set $t-5$ arbitrary elements from $X^{\prime}$, and again we get a set of size $t$ that does not admit a good coloring. Therefore $k \geq \frac{1}{2} \cdot n^{1 / 2}(1-o(1))$ and the lemma follows.

We proceed with the proofs of the remaining items of Theorem 1.5.

Proof of Item 2 in Theorem 1.5. Let $K_{n}$ be the complete graph on $n$ vertices, and suppose for simplicity that $n$ is even. Let $\left(v_{1}, v_{2}\right),\left(v_{3}, v_{4}\right), \ldots$, $\left(v_{n-1}, v_{n}\right)$ be $n / 2$ independent edges. Fix a set of colorings $f_{v_{1}}, \ldots, f_{v_{n}}$ with $k$ colors such that for every copy of $I_{t}$ there is a vertex in that copy that assigns distinct colors to all edges of this copy. Consider the complete hypergraph $K_{n / 2}^{(t)}$, where each vertex is associated with one of the $n / 2$ independent edges. Every vertex $x \in V\left(K_{n / 2}^{(t)}\right)$ that corresponds to an edge $\left(v_{2 i-1}, v_{2 i}\right)$ defines a coloring of $V\left(K_{n / 2}^{(t)}\right)$ with $k^{2}$ colors, as the combination of $f_{v_{2 i-1}}$ and $f_{v_{2 i}}$. By the definition of local coloring, for every $e \in E\left(K_{n / 2}^{(t)}\right)$ there is $v \in e$ that gives all the vertices in $e$ distinct colors. Thus by Lemma 5.1 $k^{2} \geq n^{1 / 3}(1-o(1)$ ) (if $t=4)$ and $k^{2} \geq n^{1 / 2}\left(\frac{1}{2}-o(1)\right.$ ) (if $t \geq 5$ ), and the result follows.

Proof of Item 3 in Theorem 1.5. Fix a set of $n$ colorings of $K_{n}$ such that for every star $S_{t}$, there is a vertex that assigns distinct colors to all edges of the star. Fix an arbitrary vertex $x \in V\left(K_{n}\right)$, then by the pigeonhole principle there is a set $S \subseteq V\left(K_{n}\right)$ of size $(n-1) / k$ such that $f_{x}(x u)$ is the same for every $u \in S$. Consider the complete hypergraph $K_{(n-1) / k}^{(t)}$, where each vertex is associated with one of the vertices of $S$, and define a set of $(n-1) / k$ $k$-colorings $\left\{g_{v}: v \in S\right\}$ of the hypergraph by $g_{v}(u)=f_{v}(x u)$. By definition, for every $e \in K_{(n-1) / k}^{(t)}$ there is $v \in e$ that gives distinct colors for all the vertices of $e$, and thus by Lemma 5.1 we have $k \geq(1-o(1))\left(\frac{n}{k}\right)^{1 / 3}$ (if $t=4$ ) and $k \geq(1 / 2-o(1))\left(\frac{n}{k}\right)^{1 / 2}$ (if $\left.t \geq 5\right)$. Hence we get $k=\Omega\left(n^{1 / 4}\right)$ in the first case and $k=\Omega\left(n^{1 / 3}\right)$ in the second case, as required.

Finally, we get a bound for the case of a path of length at least 7 as an immediate corollary.

Proof of Item 4 in Theorem 1.5. Every path of length $t$ contains a spanning subgraph consisting of $\lceil t / 2\rceil$ independent edges. Therefore, the desired result follows from Item 2. 
Proof of Theorem 1.6. By Vizing's Theorem, any graph $H$ with at least 13 edges contains either a vertex of degree at least 4 or 4 independent edges. Therefore, it contains a spanning subgraph consisting of a vertex of degree 4 and a set of some $r$ isolated vertices, or a spanning subgraph consisting of 4 independent edges and a set of $q$ isolated vertices. In the first case we fix $r$ vertices $v_{1}, \ldots, v_{r}$ and another vertex $x$, and apply the pigeonhole principle to find a set of $(n-r-1) / k^{r}$ vertices $u_{j} \neq\left\{x, v_{1}, \ldots v_{r}\right\}$, so that for each vertex $v_{i}, f_{v_{i}}\left(x u_{j}\right)$ is constant for all $u_{j}$. We then apply the argument in the proof of Item 3 to the star consisting of all edges $x u_{j}$. The second case is similar. We first fix $q$ vertices $v_{i}$ and find a set of $\frac{n-q}{2 k^{q}}$ independent edges on the other vertices that are colored the same by each vertex $v_{i}$. We then apply the argument in the proof of Item 2 to this set.

\section{Concluding remarks and open questions}

We introduced the notion of $(n, H)$-local colorings and studied various general and concrete bounds on $C(n, H)$-the minimum number of colors that are required such that there are $n$ colorings of $K_{n}$ so that for every copy of $H$, one of the vertices assigns distinct colors to all edges of that copy. This generalizes and partially answers a question of Karchmer and Wigderson.

We characterized the (small) family of graphs that require a constant number of colors. It would be interesting to decide whether every graph apart from the members of this set requires a polynomial number of colors. We can strengthen the assertion of Theorem 1.6 and show that it holds with the same $b$ for every $H$. More precisely, there are absolute positive constants $b$ and $c$ such that for any graph $H$ with at least $c$ edges there is a constant $a(H)>0$ so that $C(n, H) \geq a(H) n^{b}$ for every $n$. We omit the detailed argument.

The problem of improving the lower bound for the case of $H=P_{3}$ is interesting as well, and will improve the lower bound for the KarchmerWigderson question. Also, it would be nice to obtain better upper bounds by exhibiting (probabilistic or explicit) colorings.

Finally, there are gaps between the upper and lower bounds of $C(n, H)$ for almost every $H$, and closing the gap in each of the considered cases might need additional ideas. In particular, it would be nice to decide if for every $\varepsilon>0$, there is an $r=r(\varepsilon)$ so that $C\left(n, K_{r}\right) \geq n^{1-\varepsilon}$ for all sufficiently large $n$. 


\section{References}

[1] Alon, N. and Spencer, J. (2008). The Probabilistic Method, 3rd ed. Wiley. MR2437651

[2] Bondy, J. A. and Murty, U. S. R. (2008). Graph Theory, 3rd ed. Springer. MR2368647

[3] Erdős, P. and Rado, R. (1952). Combinatorial theorems on classifications of subsets of a given set. Proc. London Math. Soc. 2(3) 417-439. MR0065615

[4] Jukna, S. (2001). Extremal Combinatorics With Applications in Computer Science, Springer. MR1931142

[5] Karchmer, M. and Wigderson, A. (1993). On span programs. In: Proc. of The 8th Annual Symposium on Structure in Complexity, pp. 102-111. MR1310791

[6] Wigderson, A. (1993). The fusion method for lower bounds in circuit complexity. In: Combinatorics, Paul Erdös is Eighty (Vol. 1), D. Miklós, V. T. Sós and T. Szönyi (Eds.), Bolyai Math. Society, pp. 453-468. MR1249727

Noga AlON

SACKLER School of Mathematics

and Blavatnik School of Computer Science

Tel Aviv University

TEL Aviv 69978

ISRAEL

Institute for Advanced Study

Princeton, New Jersey, 08540

USA

E-mail address: nogaa@tau.ac.il

IDO BEN-ELIEZER

Blavatnik School of Computer Science

Tel Aviv University

Tel Aviv 69978

ISRAEL

E-mail address: idobene@post.tau.ac.il

Received April 13, 2011 